\title{
Characterization of locally dually flat Matsumoto metric
}

\author{
Narasimhamurthy S.K, Kavyashree A.R and Mallikarjun Y.Kumbar \\ Department of P.G. Studies and Research in Mathematics, Kuvempu University, Shankaraghatta - 577451, \\ Shimoga, Karnataka, INDIA.
}

\begin{abstract}
The concept of locally dually flat Finsler metrics are originated from information geometry. As we know, $(\alpha, \beta)$-metrics defined by a Riemannian metric $\alpha$ and 1-form $\beta$ form an important class of Finsler metrics. In this paper, we study and characterize Locally dually flat Matsumoto metric $F=\alpha^{2} / \alpha-\beta$ with isotropic S-curvature which is not Riemannian.
\end{abstract}

Key words: Matsumoto metric, locally dually flat, locally minkowskian, isotropic S-curvature.

AMS Subject Classification (2010): 53C60, 53B40

\section{Introduction}

The notion of dually flat metrics was first introduced by Amari S.I and H.Nagaoka when they study the information geometry on Riemannian spaces [1]. Later Z.Shen extends the notion of dually flatness to Finsler metrics [8]. Dually flat Finsler metrics form a special and valuable class of Finsler metrics in Finsler information geometry, which play a very important role in studying flat Finsler information structure ([3],[4],[10],[11],[12]).

In 2009, the authors [3] classified the locally dual flat Randers metrics with almost isotropic flag curvature. Recently, Xia.Q worked on the dual flatness of Finsler metrics of isotropic flag curvature as well as scalar flag curvature ([11],[12]). Also, Xia studied and gave a characterization of locally dually flat $(\alpha, \beta)$ metrics on an $n$-dimensional manifold $\mathrm{M}(n \geq 3)[10]$.

The first example of non-Riemannian dually flat metrics is the Funk metric given as follows([3],[8]):

$$
F=\frac{\sqrt{\left(1-|x|^{2}\right)|y|^{2}+\langle X, Y\rangle^{2}}}{1-|x|^{2}} \pm \frac{\langle X, Y\rangle}{1-|x|^{2}}
$$

This metric is defined on the unit ball $B_{n} \subset R_{n}$ and is a Randers metric with constant flag Curvature $K=-\frac{1}{4}$. This is only known example of locally dually flat metrics with non-zero constant flag curvature up to now.

In this paper, we study and characterize locally dually flat Matsumoto metric with isotropic S-curvature which is not Riemannian.

\section{Preliminaries}

Let $M$ be an $n$-dimensional smooth manifold. We denote by $T M$ the tangent bundle of $M$ and by $(x, y)=\left(x^{i}, y^{i}\right)$ the local coordinates on the tangent bundle TM. A Finlser manifold $(M, F)$ is a smooth manifold equipped with a function $F: T M \rightarrow[0, \infty)$ which has the following properties:

- Regularity: $F$ is smooth in $T M \backslash\{0\}$;

- Positively homogeneity: $F(x, \lambda y)=\lambda F(x, y)$, for $\lambda>0$;

- Strong convexity: the Hessian matrix of $F^{2}, g_{i j}(x, y)=\frac{1}{2}\left(\frac{\partial^{2} F^{2}(x, y)}{\partial y^{i} \partial x^{i}}\right)$, , is positive definite on $T M \backslash\{0\}$. We call $F$ and the tensor $g_{i j}$ the Finsler metric and fundamental tensor of $M$ respectively.

For a Finsler metric $F=F(x, y)$, its geodesics curves are characterized by the system of differential equations $\ddot{c}^{i}+2 G^{i}(\ddot{c})=0$, where the local functions $G^{i}=G^{i}(x, y)$ are called the spray coefficients and given by following

$$
G^{i}=\frac{1}{4} g^{i l}\left\{\frac{\partial^{2}\left[F^{2}\right]}{\partial x^{k} \partial y^{l}} y^{k}-\frac{\partial\left[F^{2}\right]}{\partial x^{l}}\right\}, \quad y \in T_{x} M .
$$

Definition 2.1: A Finsler metric $F=F(x, y)$ on a manifold $M$ is said to be locally duallyflat if at any point there is a standard coordinate system $\left(x^{i}, y^{i}\right)$ in TM which satisfies

$$
\left(F^{2}\right)_{x^{k} y^{l}}=2\left(F^{2}\right)_{x^{l}}
$$

In this case, the coordinate $\left(x^{i}\right)$ is called an adapted local coordinate system. It is easy to see that every locally Minkowskian metric is locally dually flat. But the converse is not true[3]. 
Definition 2.2: A Finsler metric is said to be locally projectively flat if at any point there is a local coordinate system in which the geodesics are straight lines as point sets.

It is known that a Finsler metric $F(x, y)$ on an open domain $U \subset \mathbb{R}^{n}$ is locally projectively flat if and only if its geodesic coefficients $G^{i}$ are in the form

$$
G^{i}=P y^{i}
$$

where $P: T U=U \times \mathbb{R}^{n} \rightarrow \mathbb{R}$ is positively homogeneous with degree one, $P(x, \lambda y)=\lambda P(x, y), \lambda>0$. We call $P(x, y)$ the projective factor of $F(x, y)$.

Lemma 2.1. [3] Let $F=F(x, y)$ be a Finsler metric on an open subset $U \subset \mathbb{R}^{n}$. Then $F$ is locally flat and projectively flat on $U$ if and only if $F_{x^{k}}=C F F_{y^{k}}$, where $C$ is a constant.

The S-curvature is a scalar function on $T M$, which was introduced by the Shen to study volume comparison in Riemann-Finsler geometry [9]. The S-curvature measures the average rate of change of $\left(T_{x} M, F_{x}=\left.F\right|_{T_{x} M}\right)$ in the direction $y \in T_{x} M$. It is known that $S=0$ for Berwald metrics.

Definition 2.3: A Finsler metric $F$ on an n-dimensional manifold $M$ is said to have isotropic $S$-curvature if isotropic $S=(n+1) c(x) F$, for some scalar function $c$ on $M$.

For a Finsler metric $F$ on an $n$-dimensional manifold $M$, the Busemann-Hausdorff volume form $d V_{F}=\sigma_{F}(x) d x^{1} \ldots \ldots \ldots d x^{n}$ is defined by

$$
\sigma(F)=\frac{\operatorname{Vol}\left(B^{n}(1)\right)}{\operatorname{Vol}\left\{\left(y^{i}\right) \in \mathbb{R}^{n} \mid F\left(\left.y^{i} \frac{\partial}{\partial x^{i}}\right|_{x}\right)\right\}}
$$

Here $V o l$ denotes the Euclidean volumes and $B^{n}(1)$ denotes the unit ball in $\mathbb{R}^{n}$. Then the S-curvature is defined by

where $y=\left.y^{i} \frac{\partial}{\partial x^{i}}\right|_{x} \in T_{x} M[9]$.

$$
S(y)=\frac{\partial G^{i}}{\partial y^{i}}(x, y)-y^{i} \frac{\partial}{\partial x^{i}}\left[\ln \sigma_{F}(x)\right]
$$

For an $(\alpha, \beta)$-metric $F=\alpha \phi(s)$ where $s=\beta / \alpha$ and $\phi=\phi(s)$ is a $C^{\infty}$ function on the $\left(-b_{0}, b_{0}\right)$ with certain regularity, $\alpha=\sqrt{a_{i j} y^{i} y^{j}}$ is a Riemannian metric and $\beta=b_{i}(x) y^{i}$ is 1 -form on $\mathrm{M}$.

Let us define $b_{i \mid j}$ by

$$
b_{i \mid j} \theta^{j}=d b_{i}-b_{j} \theta_{i}^{j}
$$

where $\theta^{i}=d x^{i}$ and $\theta_{i}^{j}=\Gamma_{i k}^{j} d x^{k}$ denote the Levi- Civita connection form of $\alpha$. Let

$$
r_{i j}=\frac{1}{2}\left(b_{i \mid j}+b_{j \mid i}\right), \quad s_{i j}=\frac{1}{2}\left(b_{i \mid j}-b_{j \mid i}\right) .
$$

Clearly, $\beta$ is closed if and only if $s_{i j}=0$. An $(\alpha, \beta)$-metric is said to be trivial if $r_{i j}=s_{i j}=0$. put

$$
\begin{gathered}
r_{i 0}=r_{i j} y^{j}, \quad r_{00}=r_{i j} y^{i} y^{j}, \quad r_{j}=b^{i} r_{i j}, \\
s_{i 0}=s_{i j} y^{j}, \quad s_{j}=b^{i} s_{i j}, \\
r_{0}=r_{j} y^{j}, \quad s_{0}=s_{j} y^{j} .
\end{gathered}
$$

By a direct computation, we can obtain a formula for mean Cartan torsion of an $(\alpha, \beta)$-metric as follows

$$
I_{i}=-\frac{\Phi(\phi-s \phi)}{2 \Delta \phi \alpha^{2}}\left(\alpha b_{i}-s y^{i}\right)
$$

Clearly, an $(\alpha, \beta)$-metric $F=\alpha \phi(s)$ where $s=\beta / \alpha$ is Riemannian if and only if $\Phi=0$. Hence, further we suppose that $\Phi \neq 0$.

Theorem 2.1. [10] Let $F=\alpha \phi(s), s=\beta / \alpha$ be an $(\alpha, \beta)$-metric on an $n$-dimensional manifold $M_{n}(n \geq 3)$ where $\alpha=\sqrt{a_{i j} y^{i} y^{j}}$ is a Riemannian metric and $\beta=b_{i}(x) y^{i}$ is a 1-form on M. Suppose that $F$ is not Riemannian and $\phi^{\prime}(s) \neq 0$. Then $F$ is locally dually flat on $M$ if and only if $\alpha, \beta$ and $\phi=\phi(s)$ satisfy 
1. $s_{l 0}=\frac{1}{3}\left(\beta \theta_{l}-\theta b_{l}\right)$,

2. $r_{00}=\frac{2}{3} \theta \beta+\left[\tau+\frac{2}{3}\left(b^{2} \tau-\theta_{l} b^{l}\right)\right] \alpha^{2}+\frac{1}{3}\left(3 k_{2}-2-3 k_{3} b^{2}\right) \tau \beta^{2}$,

3. $G_{\alpha}^{l}=\frac{1}{3}\left[2 \theta+\left(3 k_{1}-2\right) \tau \beta\right] y^{l}+\frac{1}{3}\left(\theta^{l} \tau b^{l}\right) \alpha^{2}+\frac{1}{2} k_{3} \tau \beta^{2} b^{l}$,

$\tau\left[s\left(k_{2}-k_{3} s^{2}\right)\left(\phi \phi^{\prime}-s \phi^{2}-s \phi \phi^{\prime \prime}\right)-\left(\phi^{2}+\phi \phi^{\prime \prime}\right)+k_{1} \phi\left(\phi-s \phi^{\prime}\right)\right]=0$,

where $\tau=\tau(x)$ is a scalar function, $\theta=\theta_{i}(x) y^{i}$ is a l-form on $M$ and $\theta^{l}=a^{l m} \theta_{m}$ and

$k_{1}=\Pi(0), k_{2}=\frac{\Pi^{\prime}(0)}{Q(0)}$

$k_{3}=\frac{1}{6 Q(0)^{2}}\left[3 Q^{\prime \prime}(0) \Pi^{\prime}(0)-6 \Pi(0)^{2}-Q(0) \Pi^{\prime \prime \prime}(0)\right]$,

$Q=\frac{\phi^{\prime}}{\phi-s \phi^{\prime}}, \Pi=\frac{\phi^{2}+\phi \phi^{\prime \prime}}{\phi\left(\phi-s \phi^{\prime}\right)}$.

In [5], Cheng-Shen study the class of $(\alpha, \beta)$-metrics of non-Randers type $\phi \neq t_{1} \sqrt{1+t_{2} s^{2}}+t_{3} s$ with isotropic S-curvature and obtain the following.

Theorem 2.2. [5] Let $F=\alpha \phi(s), s=\beta / \alpha$ be an non-Riemannian $(\alpha, \beta)$-metric on a manifold and $b=\left\|\beta_{x}\right\|_{\alpha}$. Suppose that $\phi \neq t_{1} \sqrt{1+t_{2} s^{2}}+t_{3} s$ for any constant $t_{1}>0, t_{2}$ and $t_{3}$. Then $F$ is of isotropic $S$-curvature $S=$ $(n+1) c F$, if and only if one of the following holds

i) $\beta$ satisfies

$$
r_{i j}=\varepsilon\left\{b^{2} a_{i j}-b_{i} b_{j}\right\}, s_{j}=0,
$$

where $\varepsilon=\varepsilon(x)$ is a scalar function, and $c=c(x)$ satisfies

$$
\Phi=-2(n+1) k \frac{\phi \Delta^{2}}{b^{2}-s^{2}},
$$

where $k$ is a constant. In this case, $S=(n+1) c F$ with $c=k \varepsilon$.

ii) $\beta$ satisfies

$$
r_{i j}=0, s_{j}=0 .
$$

In this case, $S=0$, regardless of choices of a particular $\phi$.

\section{Characterization of locally dually flat Matsumoto metric}

Theorem 3.3. Let $F=\alpha^{2} / \alpha-\beta$ be a Matsumoto metric on a manifold $M$ of dimension $n \geq 3$. Then the necessary and sufficiency conditions for $F$ to be locally dually flat on $M$ is as follows:

i) $s_{l 0}=\frac{1}{3}\left(\beta \theta_{l}-\theta b_{l}\right)$,

ii) $r_{00}=\frac{2}{3} \theta \beta+\left[\tau+\frac{2}{3}\left(b^{2} \tau-\theta_{l} b^{l}\right)\right] \alpha^{2}+\frac{26}{3} \tau \beta^{2}$,

iii) $G_{\alpha}^{l}=\frac{1}{3}[2 \theta+7 \tau \beta] y^{l}+\frac{1}{3}\left(\theta^{l}-\tau b^{l}\right) \alpha^{2}$,

where $\tau=\tau(x)$ is a scalar function and $\theta=\theta_{k} y^{k}$ is a 1-form on $M$.

Proof: For a Matsumoto metric $F=\alpha^{2} / \alpha-\beta$, we obtain the following as

$$
\begin{array}{ll}
\phi=\frac{1}{1-s}, & \phi^{\prime}=\frac{1}{(1-s)^{2}}, \\
Q=\frac{1}{1-2 s}, & Q^{\prime}=\frac{2}{(1-2 s)^{2}}, \\
Q^{\prime \prime}=\frac{8}{(1-2 s)^{3}}, & Q^{\prime \prime}=\frac{48}{(1-2 s)^{3}}, \\
\Pi=\frac{3}{1-3 s+2 s^{2}}, & \Pi^{\prime}=\frac{-3(4 s-3)}{\left(1-3 s+2 s^{2}\right)^{2}}, \\
\Pi^{\prime \prime}=\frac{-6\left(12 s^{2}-18 s+7\right)}{\left(1-3 s+2 s^{2}\right)^{3}}, & \Pi^{\prime \prime \prime}=\frac{18\left(8 s^{2}-12 s+5\right)(4 s-3)}{\left(1-3 s+2 s^{2}\right)^{4}}, \\
k_{1}=3, k_{2}=9 \text { and } k_{3}=0 .
\end{array}
$$

By using above values in Theorem 2.1, we get

$\tau\left[s\left(k_{2}-k_{3} s^{2}\right)\left(\phi \phi^{\prime}-s \phi^{2}-s \phi \phi^{\prime \prime}\right)-\left(\phi^{2}+\phi \phi^{\prime \prime}\right)+k_{1} \phi\left(\phi-s \phi^{\prime}\right)\right]=0$ and $\tau=0$.

Then finally by substituting $k_{1}, k_{2}$ and $k_{3}$ values in Theorem 2.1 , we get required proof.

Now, let $\phi=\phi(s)$ be a $C^{\infty}$ function on the $\left(-b_{0}, b_{0}\right)$. For a number $b \in\left[0, b_{0}\right]$ let

$$
\Phi=-\left(Q-s Q^{\prime}\right)\{n \Delta+1+s Q\}-\left(b^{2}-s^{2}\right)(1+s Q) Q^{\prime \prime},
$$


where $\Delta=1+\mathrm{s} Q+\left(b^{2}-s^{2}\right) Q^{\prime}$.

It implies that

$\Delta=\frac{1+2 b^{2}-3 s}{(1-2 s)^{2}}$.

By $Q$, the the equation (3.1) can be written as follows:

$$
\Phi=-\left(Q-s Q^{\prime}\right)(n+1) \Delta+\left(b^{2}-s^{2}\right)\left\{\left(Q-s Q^{\prime}\right) Q^{\prime}-(1+s Q) Q^{\prime \prime}\right\}
$$
curvature.

By using the Theorem 2.2, now we will consider locally dually flat $(\alpha, \beta)$-metric with isotropic $S$ -

Theorem 3.4. Let $F=\alpha^{2} / \alpha-\beta$ be a locally dually flat non-Randers type $(\alpha, \beta)$-metric on a manifold $M$ of dimension $n \geq 3$. Suppose that $F$ is of isotropic $S$-curvature $S=(n+1) c F$, where $c=c(x)$ is a scalar function on $M$. Then $F$ is a locally projectively flat in adapted coordinate system and $G^{i}=0$.

Proof: Let $G^{i}=G^{i}(x, y)$ and $\bar{G}_{\alpha}^{i}=\bar{G}_{\alpha}^{i}(x, y)$ denote the coefficients of $F$ and $\alpha$ respectively in the same coordinate system.

By definition, we have

where

$$
G^{i}=\bar{G}_{\alpha}^{i}+P y^{i}+Q^{i},
$$

$$
\begin{aligned}
P & =\alpha^{-1} \Theta-2 Q \alpha s_{0}+r_{00}, \\
Q^{i} & =\alpha Q s_{0}^{i}+\Psi-2 Q \alpha s_{0}+r_{00} b^{i}, \\
\Theta & =\frac{\phi \phi^{\prime}-s\left(\phi \phi^{\prime \prime}+\phi^{\prime} \phi\right)}{2 \phi\left(\left(\phi-s \phi^{\prime}\right)+\left(b^{2}-s^{2}\right) \phi^{\prime \prime}\right)} \\
\Psi & =\frac{1}{2} \frac{\phi^{\prime \prime}}{(\phi-s \phi)+\left(b^{2}-s^{2}\right) \phi^{\prime \prime}}
\end{aligned}
$$

First, we suppose that the case (i) of the Theorem 2.2 holds. It is remarkable that, for a Matsumoto metric,

$$
\Delta=\frac{1+2 b^{2}-3 s}{(1-2 s)^{2}} .
$$

It follows that $(1-2 s)^{2}$ is a polynomial in $\mathrm{s}$ of degree 3 . On the other hand we have

$$
\phi \Delta^{2}=\frac{\phi\left(1+2 b^{2}-3 s\right)^{2}}{(1-2 s)^{4}} .
$$

Hence, if case(ii) of theorem (2.2) holds, then substituting (3.7) we obtained that

$$
\left(b^{2}-s^{2}\right)(1-2 s)^{4} \Phi=-2(n+1) k \phi\left(1+2 b^{2}-3 s\right)^{2} .
$$

It follows that $\left(b^{2}-s^{2}\right)(1-2 s)^{4} \Phi$ is not a polynomial in s (if $k=0$, then by considering the cartan torsion equation, we get a contradiction).

Then, we put

where

$$
\phi \Delta^{2}=\frac{\bar{\Delta}}{(1-2 s)^{4}}
$$

$$
\bar{\Delta}=\phi\left(1+2 b^{2}-3 s\right)^{2}=\frac{\left(1+2 b^{2}-3 s\right)}{1-2 s} .
$$

By assumption $F$ is a non-Randers type metric. Thus $\bar{\Delta}$ is not a polynomial in $\mathrm{s}$ and then $\left(b^{2}-s^{2}\right)(1-2 s)^{4} \Phi$ is not a polynomial in s. Now, let us consider another formula for $\Phi$ :

$$
\Phi=-\left(Q-s Q^{\prime}\right)(n+1) \Delta+\left(b^{2}-s^{2}\right)\left\{\left(Q-s Q^{\prime}\right) Q^{\prime}-(1+s Q) Q^{\prime \prime}\right\},
$$

where

$$
\left(Q-s Q^{\prime}\right)=\frac{1-4 s}{(1-2 s)^{2}} .
$$

Then

$$
\Phi=\frac{(n+1)(1-4 s)\left(1+2 b^{2}-3 s\right)+\left(b^{2}-s^{2}\right)(1-7 s)}{(1-2 s)^{4}} .
$$

From equations (3.8) and (3.11) the relation $\left(b^{2}-s^{2}\right)(1-2 s)^{4} \Phi$ is a polynomial in $s$ and $b$ of degree 5 and 4 respectively. The coefficient of $s^{5}$ is 7 which is not equal to zero $(7 \neq 0)$. Hence its impossible that $\Phi=0$. Therefore, we can conclude that equation (2.3) does not hold. So, the case (ii) of the Theorem 2.2 holds. In this case, we have 


$$
\begin{gathered}
r_{00}=0, \\
s_{j}=0 .
\end{gathered}
$$

In Theorem3.3(ii), taking $r_{00}=0$ and we obtain

$$
\left[\tau+\frac{2}{3}\left(b^{2} \tau-\theta^{m} b_{m}\right)\right] \alpha^{2}=\beta\left[-\frac{2}{3} \theta-\frac{25}{3} \beta \tau\right] .
$$

Since $\alpha^{2}$ is irreducible polynomial of $y^{i}$, the equation (3.14) reduces to the following

$$
\begin{aligned}
\tau+\frac{2}{3}\left(b^{2} \tau-b_{m} \theta^{m}\right), & \\
\frac{2}{3} \theta+\frac{25}{3} \beta \tau & =0 .
\end{aligned}
$$

Then Theorem 3.3(i) becomes

$$
s_{i 0}=-\frac{1}{3}\left(\theta b^{2}-\beta b_{m} \theta^{m}\right) \text {. }
$$

This implies that

$$
\theta b^{2}-\beta b_{m}=0
$$

By equations (3.14), (3.15) and (3.16), we obtain

$$
-\frac{2}{3}\left(1+2 b^{2}\right)=\tau+\frac{2}{3}\left(b^{2}-b_{m} \theta^{m}\right) \beta+\frac{25}{3}\left(1+2 b^{2}\right) \tau \beta .
$$

It implies that

$$
\theta=-\frac{25}{3} \tau \beta \text {. }
$$

From equations (3.15) and (3.20), it follows that $\tau=0$ and substituting $\tau=0$ in equation (3.20) we get $\theta=0$. Thus finally Theorem 2.1 (i),(ii) and (iii) reduce to the following

$$
\begin{aligned}
& s_{i j}=0, \\
& G_{\alpha}^{l}=0, \\
& r_{00}=0 .
\end{aligned}
$$

Since $s_{0}=r_{00}=0$, then equations (3.5) and (3.6) reduces to

$$
P=0 \text { and } Q^{i}=0 \text {. }
$$

Then the relation (3.4) becomes

$$
G_{\alpha}^{i}=0 .
$$

This completes the proof.

Theorem 3.5. Let $F=\alpha^{2} / \alpha-\beta$ be a Matsumoto metric and non-Riemannian metric on $n$-dimensional $(n \geq 3)$ manifold $M$. Then $F$ is locally dually flat with isotropic $S$-curvature. $S=(n+1) c F$ if and only if it is locally Minkowskian.

Proof: We concluded by the theorem 3.4 that $F=\alpha^{2} / \alpha-\beta$ is dually flat and projectively flat in any adapted coordinate system. By lemma 2.1 , we have

Hence the spray coefficients $G^{i}=P y^{i}$ are given by

$$
F_{x^{k}}=C F F_{y^{k}} .
$$

Since $G^{i}=0$, then $P=0$ and so that $C=0$.

$$
P=\frac{1}{2} C F
$$

It implies that $F_{x^{k}}=0$ and then $F$ is a locally Minkowskian metric in the adapted coordinated system. This completes the proof.

\section{Conclusion}

The authors S.I.Amari and H.Nagaoka[1] introduced the notion of dually flat Riemannian metrics when they study the information geometry on Riemannian manifolds. Information geometry has emerged from investigating the geometrical structure of a family of probability distributions and has been applied successfully to various areas including statistical inference, control system theorem and multi-terminal information theorem.

As we know, Finsler geometry is just Riemannian geometry without the quadratic restriction. Therefore, it is natural to extending the construction of locally dually flat metrics for Finsler geometry. 
In Finsler geometry, Z.Shen [8] extends the notion of locally dually flatness for Finsler metrics. Dually flat Finsler metric form a special and valuable class of Finsler metric in Finsler information geometry, which play a very important role in studying many applications in Finsler information structure.

In this article, we study and characterized the locally dually flat Matsumoto metric with isotropic Scurvature which is not Riemannian.

\section{References}

[1] S.I. Amari and H.Nagaoka, Methods of information geometry-Oxford University Press, AMS Translation of Math. Monographs, $191,(2000)$

[2] S. Bacso, X. Cheng and Z. Shen, Curvature Properties of $(\alpha, \beta)$--metrics, Adv. Stud. Pure Math. Soc., Japan (2007),

[3] Cheng,X., Shen. Z and Zhou, On a class of Locally dually flat Finsler metrics. J.Math., 21(11), 1-13(2005).

[4] X. Cheng and Tian.Y, Locally dually flat Finsler metrics with special curvature properties., J.Diff.Geom.Appl. 2011.

[5] X. Cheng and Z. Shen, A Class of Finsler metrics with isotropic S-curvature, Israel J. Math., 169(2009), $317-340$.

[6] S.K.Narasimhamurthy, Latha Kumari G.N and C.S.Bagewadi, Projectively flat kropina metric with $K=0$. J. Applied Mathematical Sciences., 5(2011), 585-593.

[7] S.K.Narasimhamurthy and D.M.Vasantha, Projective change between Randers metric and Special ( $\alpha, \beta)$-metrics sJ. Informatics and Mathematical Sciences, (2012).

[8] Z. Shen, Riemann-Finsler geometry with applications to information geometry, J. Chin. Ann.Math., 27B(1)(2006), 73-94.

[9] Z. Shen, Volume Comparison and its applications in Riemann-Finsler Geometry, J. Adv. in Math.,128 (1997), $306-328$.

[10] Xia,Q., On locally dually flat $(\alpha, \beta)$-metrics J. Differential geometry and applications, 29(2), (2011),233-243.

[11] Xia.Q, On a class of locally dually flat Finsler metrics of isotropic flag curvature, J.Publ.Math. Debrcen 78(1), (2011), 169-190.

[12] Xia.Q, On a class of locally dually flat Finsler metrics of scalar flag curvature, J.Publ.Math. Debrcen in Press. 